\title{
Valoración del alumnado sobre el uso de Clickers y vídeo tutoriales en educación superior
}

\author{
Student Appraisal of the Use of Clickers and Video Tutorials in Higher Education \\ Valoració de l'alumnat sobre l'ús de clickers i vídeo tutorials en l'educació superior
}

\author{
María Isabel López-Rodríguez ${ }^{*}$, Maja Barac ${ }^{\circledR}$ \\ Departamento de Economía Aplicada, Universitat de València, Valencia, Comunidad Valenciana, España \\ *Autor para correspondencia: maria.i.lopez@uv.es (María Isabel López-Rodríguez)
}

Recibido: 21/11/2018 | Aceptado: 27/12/2018 | Publicado: 24/06/2019

\section{Cómo citar: \\ López-Rodríguez, M. I., y \\ Barac. M. (2019). \\ Valoración del alumnado sobre el uso de Clickers y vídeo tutoriales en educación superior. Research in Education and Learning Innovation Archives, 22,29-44. 10.7203/realia.22.14582}

Copyright: El/La Autor/a. Open Access: Este es un artículo de acceso abierto distribuido bajo los términos de la licencia Creative Commons AttributionNonCommercial-ShareAlike 4.0 International (CC BY-NC-SA 4.0)

Financiación: Projecte d'Innovació Educativa i Qualitat Docent: Elaboració de materials interactius $i$ multidisciplinaris per afavorir l'aprenentatge i avaluació en els estudis de grau

(UV-SFPIE_GER17-585541)
RESUMEN: La puesta en marcha de los grados y postgrados en las universidades españolas ha venido acompañada de una revisión de las metodologías docentes utilizadas y la incorporación en muchos casos de Tecnologías de la Información y la Comunicación (TIC). Tecnologías como las Herramientas de Respuesta de Audiencia (HRA), los Clickers, y los vídeos (mUVies) se utilizaron en una asignatura cuantitativa de un grado ofertado por la Universitat de València (UV), en un intento de paliar la baja carga lectiva de la misma y dotar al alumnado de material de apoyo dentro y fuera del aula. El trabajo que se presenta examina la percepción que el alumnado tuvo del uso de dichas TIC en su proceso de aprendizaje y también el volumen de trabajo que supuso su puesta en marcha. Utilizando análisis descriptivos e inferenciales sobre las opiniones obtenidas en las encuestas acerca de las TIC y los resultados de la evaluación continua y el examen final, concluimos que el alumnado valoró muy positivamente el uso de ambas TIC con una calificación media superior a 8 (sobre 10), valorando mejor los mUVies que los Clickers. En cuanto al volumen de trabajo que la implantación de las TIC supuso, esta se estima en unas 7.5 horas para el alumnado y de unas 132.5 horas para el profesorado.

PALABRAS CLAVE: tecnología educativa; mUVies; educación superior; grado en International Business

ABSTRACT: The implementation of undergraduate and postgraduate degrees in Spanish universities has been accompanied by a review of the teaching methodologies used. These methodologies incorporating, in many cases, new Information and Communication Technologies (ICT). The ICT of Audience Response Systems (ARS), Clickers, and videos (mUVies) were used in a quantitative subject of a degree offered by the Universitat de València (UV), in an attempt to mitigate the low teaching load of the same and provide students with support material inside and outside the classroom. The work presented examines the students' perception of the use of these ICTs in their learning process and also the volume of work involved in its implementation. Using descriptive and inferential analyses of the opinions obtained in the ICT surveys and the results of the continuous assessment and the final exam, we conclude that the students valued the use of both ICTs very positively with average score higher than 8 (out of 10), rating better the mUVies than Clickers. As for the volume of work that the implementation of ICTs, this is estimated at about 7.5 hours for students and about 132.5 hours for teachers.

KEYWORDS: educational technologies; mUVies; higher education; degree in International Business

RESUM: L'engegada dels graus i postgraus en les universitats espanyoles ha vingut acompanyada d'una revisió de les metodologies docents utilitzades incorporant, en molts casos, l'ús de Tecnologies 
de la Informació i la Comunicació (TIC). Les TIC de Eines de Resposta d'Audiència (ERA), els Clickers, i els vídeos (mUVies) es van utilitzar en una assignatura quantitativa d'un grau de la Universitat de València (UV) en un intent de pal-liar la baixa càrrega lectiva de la mateixa i dotar a l'alumnat de material de suport dins i fora de l'aula. El treball que es presenta examina la percepció que l'alumnat tingué de l'ús d'aquestes TIC en el seu procés d'aprenentatge i també el volum de treball que suposà la seua engegada. Utilitzant anàlisis descriptives i inferencials sobre les opinions obtingudes en les enquestes sobre les TIC i els resultats de l'avaluació contínua i l'examen final, concloem que l'alumnat va valorar molt positivament l'ús d'ambdues TIC amb una qualificació mitjana superior a 8 (sobre 10), valorant millor els mUVies que els Clickers. Quant al volum de treball que la implantació de les TIC suposà, aquesta s'estima en unes 7.5 hores per a l'alumnat i d'unes 132.5 hores per al professorat.

PARAULES CLAU: tecnologies educatives; mUVies; educació superior; grau en International Business

Notas de aplicación práctica

\section{Qué se sabe sobre este tema}

- Son múltiples los trabajos de investigación cuyo objetivo se ha centrado en analizar la repercusión que el uso de las TIC y vídeos tutoriales han tenido en el output del proceso enseñanza-aprendizaje.

\section{Qué aporta este trabajo}

- El estudio se realiza desde el punto de vista que el alumnado tiene del uso de una TIC y de vídeos tutoriales, de manera que posibilita priorizar el uso de uno de ellos,

- Se analiza la posible relación entre el uso de ambas tecnologías y el rendimiento académico del alumnado, medido a través de la nota de evaluación continua y la del examen final de la asignatura,

- Se realiza un cómputo del volumen de trabajo que supone la puesta en marcha de ambas herramientas tanto para el profesorado como para el alumnado.

\section{Implicaciones para la práctica y/o política}

- Los resultados obtenidos pueden suponer una importante fuente de información para los docentes que, o bien estén sopesando la posibilidad de incorporar en la metodología que utiliza para la impartición de sus asignaturas alguna de las herramientas aquí analizadas (Clickers y mUVies) o bien, para aquellos que haciendo uso de una de ellas puedan plantearse añadir la utilización de la otra de manera combinada.

\section{INTRODUCCIÓN}

En la última década, se ha producido en las universidades españolas un cambio significativo tanto de las metodologías docentes utilizadas, como del papel de los dos agentes involucrados en el proceso de enseñanza-aprendizaje: alumnos y profesores. Así, el modelo tradicional de educación caracterizado por la clase magistral, ha dado paso a otros modelos pedagógicos en los que han irrumpido con fuerza las Tecnologías de la Información y la Comunicación (TIC). Uno de dichos modelos es el introducido por Lage, Platt, y Treglia (2000) y J. Baker (2000) bajo diferentes denominaciones y que en la actualidad se conoce como Flipped Classroom o clase invertida. Dicho modelo, que se caracteriza por un cambio de escenario de las actividades propuestas ha demostrado dar muy buenos resultados (Gross, Pietri, Anderson, Moyano-Camihort, y Graham, 2015; Pueo, Jimenez-Olmedo, Penichet-Tomas, y Carbonell-Martinez, 2017; Singh, Abdellahi, Lou-Maher, y Latulipe, 2016). En este el alumno debe invertir parte de su tiempo a adquirir fuera del aula aquellos conocimientos que necesitará para la correcta realización de las actividades dentro de esta.

Dicho cambio ha provocado un incremento notable del tiempo de elaboración de los recursos necesarios por parte del profesorado. Estos, en muchos casos, suelen ser de 
carácter audiovisual y son realizados íntegramente por el propio profesorado o bien obtenidos por este tras un proceso de filtrado, agregación y/o curación (A. Baker, 2016). Este tipo de material suele tener muy buena aceptación por parte del estudiantado, denominado por algunos autores como nativos digitales ${ }^{1}$ y que, por tanto, percibe esta herramienta como algo familiar (Perdomo, 2016; Serrano-Pastor y Casanova-López, 2017). Además, el crecimiento de los Massive Open Online Courses (MOOC) refuerza el potencial de los videos en educación (Casasús, Ivars, y López-Rodríguez, 2018; GarcíaAretio, 2017).

También ha proliferado el uso de las Herramientas de Respuesta de Audiencia (HRA), pues se ha comprobado el beneficio que suele lograr el tratamiento de preguntas dentro del aula (León, Peñalba, y Escudero, 2002). Con su uso se consigue tanto la implicación del alumnado como la obtención de un feedback inmediato por parte del profesor, que detecta en el momento de la respuesta cualquier discrepancia entre lo que se ha explicado y lo que ellos han entendido, permitiendo de esta forma una corrección inmediata (Bruff, 2009) con una repercusión positiva en el rendimiento académico tanto en estudios de grado (Barac y López-Rodríguez, 2017; Calvo-Roselló, LópezRodríguez, y Ruiz-Ponce, 2017; López-Rodríguez, Palací-López, y Palací-López, 2015) como en estudios de postgrado (López-Rodríguez y Barac, 2016; López-Rodríguez et al., 2015).

En todo caso resulta incuestionable que la incorporación de cualquiera de las tecnologías educativas existentes supone un esfuerzo añadido tanto para los docentes como para el estudiantado. Por un lado en el caso del estudiantado, este no suele conocer previamente las aplicaciones a emplear en el aula y, en el caso de conocerlas su relación con ellas no suele darse en un sentido didáctico. Además, el uso de las mismas puede incrementar su trabajo fuera o dentro del aula. Por otro lado, el esfuerzo añadido para el docente ha dado lugar a que en algunos casos se hayan propuesto modelos pedagógicos que, haciendo uso de algunas de las características y recursos propios de la clase invertida, no supongan un cambio organizativo total en la impartición de la asignatura. Así, el trabajo que se presenta refleja los resultados de la incorporación de algunas tecnologías en la impartición-asimilación de una asignatura en la que el modelo pedagógico responde a dicha situación. En efecto, ante los problemas derivados de la baja carga lectiva de una asignatura cuantitativa (Estadística) perteneciente al plan de estudios del Grado en International Business (GIB) ${ }^{2}$ ofertado por la Universitat de València (UV), el equipo docente decidió incorporar dos de las herramientas características de la clase invertida en la planificación y desarrollo de esta. Concretamente se seleccionó una HRA, y se dotó al alumnado de vídeo tutoriales docentes (denominados por la institución mUVies) elaborados por los docentes con el soporte técnico del servicio universitario correspondiente, en este caso el Servei de Formació Permanent i Innovació Educativa (SFPIE). El contenido de dichos vídeos se correspondía con las partes de la materia que resultaban más densas (es decir: distribuciones derivadas de la Normal, estimación y contrastación de hipótesis) por lo que era el propio estudiante el que decidía, atendiendo a sus necesidades, hacer uso de los mismos tantas veces como considerara. Esto indica que los vídeos constituían un material de apoyo del que el estudiantado podía hacer uso antes o después de la clase presencial.

\footnotetext{
${ }^{1}$ La denominación no está aceptada de manera generalizada. Acerca de esta cuestión puede encontrarse más información en Gallardo-Echenique (2012), donde la autora expone que dicha definición puede variar atendiendo a las sociedades, naciones, etc. y realiza una revisión de la literatura al respecto.

${ }^{2}$ La carga lectiva de la asignatura es de 4.5 créditos, siendo la única materia de contenido estadístico que el estudiante cursa en el grado. Por contra, en el resto de grados ofertados por la Facultat d'Economia, los créditos contemplados para asignaturas de similar contenido superan los 10. Esto supone que en GIB hay una disminución de créditos, en cuanto a las materias de tipo estadístico, que oscila entre el 57.14\% y el $62.5 \%$ (según titulación de referencia).
} 
Para la selección de la HRA, se tuvieron en cuenta factores tales como las prestaciones, requerimientos de uso y los resultados obtenidos por otros autores. En esta línea, Fuertes et al. (2016) realizan un estudio comparativo de las más utilizadas: Socrative, Kahoot, Poll, Everywhere, TopHat, Mentimeter, Nearpod, iClicker, Slido y Clickers y, a modo de ejemplo, se pueden mencionar los buenos resultados obtenidos mediante Socrative por Navarro-Jover y Olmo-Cazevieille (2014) y Badia-Valiente, Olmo-Cazevieille, y Navarro-Jover (2016), así como con Kahoot por Pintor-Holguín, Gargantilla-Madera, Herreros Ruiz-Valdepeñas, y López del Hierro (2014) y con los Clickers por Caballer-Tarazona y Pardo-García (2014) y Barac y Pardo-García (2015). Una vez realizado el estudio de campo, la decisión final del equipo docente fue emplear Clickers debido también a su disponibilidad en la Facultat d'Economia (UV), pero además al hecho de que era la única de las HRA analizadas que reunía las siguientes características: facilita el control de la asistencia del alumnado, posibilita que el profesor establezca el tiempo de respuesta, contempla la posibilidad de que el sondeo se realice por equipos y ofrece informes detallados de los resultados del sondeo por participante o por pregunta. Además, el software utilizado está integrado en PowerPoint, programa con el que el profesorado ya estaba familiarizado. Así, una vez seleccionada la HRA, se llevaron a cabo tres sondeos que permitieron detectar los puntos fuertes (a reforzar) y débiles (a mejorar) de la asignatura.

A raíz de lo expuesto, el objetivo del presente estudio es analizar la percepción del alumnado del uso combinado de las tecnologías mencionadas, así como el volumen de trabajo que supuso su implantación, tanto para el profesorado, como para el alumnado. Además, aunque de manera tangencial, se estudia la influencia de la metodología empleada sobre el rendimiento académico. Para lograr el objetivo propuesto se necesitaba conocer la opinión del alumnado, por lo que se elaboró un cuestionario en el que se solicitaba información relacionada con la temática en cuestión.

A partir de los datos obtenidos a través de una encuesta al estudiantado y de la información complementaria aportada por el equipo docente relativa a la carga de trabajo que supuso el uso de estas TIC, el trabajo se estructura de la siguiente forma: en el segundo epígrafe se expone la metodología utilizada, a continuación, se recogen los resultados obtenidos atendiendo a la naturaleza de las variables objeto de estudio $\mathrm{y}$, por último se muestran de manera sucinta las conclusiones relacionadas con los objetivos propuestos.

\section{METOdOLOGÍA}

\subsection{Participantes}

La implantación de las tecnologías mencionadas se llevó a cabo en el curso académico 2017-2018. El profesorado que impartió la asignatura de Estadística del grado en International Business, asignatura se imparte en el primer cuatrimestre del segundo curso, lo componían dos docentes y la matrícula del grupo ascendía a 71 estudiantes.

\subsection{Materiales}

El material elaborado para su uso mediante la HRA fue realizado en formato TurningPoint. En total se pasaron tres sondeos elaborados con preguntas tipo test sobre la materia de estadística y fueron programados para ser contrastados en el aula como parte de la evaluación continua en diferentes momentos del cuatrimestre. Los vídeos de apoyo versaban sobre las partes de la asignatura que tradicionalmente han resultado más complicadas de asimilar. Para facilitar el acceso al material se creó un espacio web dentro de la página del proyecto de innovación docente en el marco del cual se 
realizaron los materiales ${ }^{3}$. Cada sondeo constó de 10 preguntas de tipo test con 3 opciones cada una y relacionadas con la parte de la asignatura que había sido impartida hasta el momento; en el espacio web se colgaron un total de 15 vídeos que se pusieron a disposición del estudiantado.

En cuanto a la encuesta que ha permitido conocer la opinión del alumnado para valorar la utilidad del uso las herramientas TIC en la asignatura, se realizó mediante la herramienta Google Forms. Dicha encuesta de opinión constaba de tres tipos de preguntas, concretamente:

- De respuesta tipo Likert de cinco niveles desde "totalmente en desacuerdo" (1) hasta "totalmente de acuerdo" (5)

- ¿El uso de los Clickers/mUVies en clase te ha ayudado a comprender mejor los conceptos de la asignatura?

- ¿El uso de los Clickers/mUVies te ha ayudado a preparar mejor el examen final?

- ¿El uso de los Clickers contribuyó a aprovechar mejor la clase?

- ¿El uso de los Clickers hace que la clase sea más participativa?

- De valoración (escala 0 a 10) acerca del uso de ambas herramientas. Cabe indicar que dicha valoración es en sentido amplio, esto es, recoge concepciones, emociones, etc., asociadas con la experiencia del uso y relacionadas con el aprendizaje del estudiantado.

- Valora, en una escala de 0 a 10, tu experiencia con el uso de los Clickers/mUVies.

- Una tricotómica (tres posibles respuestas: 1, 2,3) acerca de número de visualizaciones de mUVies.

- Por término medio, ¿Cuántas veces te ha hecho falta visualizar el vídeo para comprenderlo?

Las respuestas proporcionadas a esta última cuestión nos permitirán analizar la repercusión que tuvo sobre el estudiantado el uso de los Clickers y los mUVies, considerando el volumen de trabajo añadido. Dicho análisis se completará obteniendo el volumen de trabajo estimado que supuso para el profesorado la creación y uso de estas tecnologías.

\subsection{Procedimiento}

Atendiendo a la estructura de la asignatura, que está formada por tres bloques claramente diferenciados: estadística descriptiva, cálculo de probabilidades e inferencia estadística, el equipo docente programó un sondeo por bloques para conocer el grado de asimilación de los contenidos del mismo. Por otra parte, se notificó al estudiantado qué vídeos debía visionar antes de la asistencia a la sesión presencial.

Así, el cronograma relativo al uso de las TIC quedó como se indica:

- Octubre: se pasó un primer sondeo con cuestiones relativas al bloque de estadística descriptiva y debían visionarse los vídeos sobre el mismo.

- Noviembre: los sondeos realizados contenían preguntas acerca de la teoría de la probabilidad y los vídeos versaban sobre la distribución Normal y las distribuciones

\footnotetext{
${ }^{3}$ El título del proyecto es "Elaboración de materiales interactivos y multidisciplinares para favorecer el aprendizaje y evaluación en los estudios de grado" (UV-SFPIE_GER17-585541) al que pertenecen las autoras de este trabajo, y los vídeos elaborados se pueden visualizar en la siguiente dirección web: http s://pages.uv.es/piclickers/cat/MenuH_mUVies.wiki
} 
derivadas de la Normal.

- Diciembre: se pasó el sondeo sobre inferencia estadística, al tiempo que debían visualizar los vídeos sobre diversas técnicas inferenciales.

Con la finalidad de valorar la opinión del estudiantado sobre las nuevas TIC introducidas en la asignatura y no influir en su respuesta, los cuestionarios de opinión fueron enviados una vez impartida la asignatura y tras calificar a los estudiantes. Contestaron un total de 28 alumnos, recogiéndose un total de 224 valoraciones. El número de participantes en la encuesta supuso una tasa de respuesta del $39.44 \%$ sobre los estudiantes matriculados en el grupo con docencia en español y del $20 \%$ sobre la matrícula de todo el grado. Creemos que esta metodología es adecuada para obtener una opinión realista de su percepción sobre la utilidad de las TIC en su aprendizaje, en términos de adecuación y de carga de trabajo.

\subsection{Análisis de los datos}

En el estudio se consideran dos tipos de variables: unas de carácter subjetivo y otras de perfil objetivo. Las primeras están relacionadas con el impacto percibido por el propio estudiantado acerca del uso de los Clickers y mUVies, mientras que las variables de tipo objetivo se refieren al rendimiento académico del estudiante, medido a través de los resultados obtenidos en la evaluación continua y en el examen final de la materia. El análisis de la información en ambos tipos de variable, se basará en el uso secuencial de herramientas estadísticas de tipo descriptivo e inferencial. Concretamente, un análisis exploratorio de los datos permitirá obtener las primeras conclusiones, mientras que el de carácter inferencial perseguirá analizar la significatividad de las relaciones obtenidas.

Las herramientas utilizadas en la fase descriptiva serán de carácter gráfico (diagrama de barras, gráfico de sectores y gráfico de Box-Whisker) y numérico (distribuciones de frecuencias), y se completarán con el cálculo de medidas de reducción adecuadas (posición, dispersión y forma). En cuanto a las herramientas consideradas en la fase inferencial, se hará uso del sesgo y curtosis estandarizados, el gráfico de probabilidad Normal y el test de Kolmogorov-Smirnov. Así, valores del sesgo y curtosis dentro del rango -2 a 2, permitirán intuir el cumplimiento de la hipótesis de Normalidad y, adicionalmente, los gráficos de probabilidad Normal posibilitarán reforzar dicha intuición, en el caso de que los datos representados no se encontraran "muy alejados" de la recta. Por último, el test de Kolmogorov-Smirnov facilitará tomar una decisión acerca del ajuste a la Normalidad para niveles de significación entre los valores asumibles para cualquier estudio estadístico (entre el $1 \%$ y el 10\%). Por otra parte, el análisis de los datos se completa con un estudio conjunto de las variables: valoración del uso de Clickers, mUVies y notas, con la finalidad de identificar posibles relaciones significativas entre ellas usando, básicamente, el coeficiente $r$ de Pearson. Con ello se pretende dar respuesta a preguntas como:

- ¿Existe relación entre una valoración positiva en el uso de los Clickers y en el uso de los mUVies desde la perspectiva de los estudiantes?

- ¿El estudiantado que proporcionó una valoración alta al uso de los Clickers/ mUVies obtuvo un buen rendimiento académico?

\section{RESULTADOS}

En el presente epígrafe se expondrán los resultados obtenidos en el análisis de las variables consideradas (subjetivas y objetivas), así como la cuantificación del volumen de trabajo que supuso para el profesorado y el alumnado el uso de las tecnologías objeto de estudio. 


\subsection{Análisis descriptivo de las variables de carácter subjetivo}

Las Figura 1 y Figura 2 reflejan los porcentajes correspondientes a las respuestas recibidas en cada uno de los niveles de las cuestiones tipo Likert relativas a la percepción del alumnado sobre Clickers y mUVies.

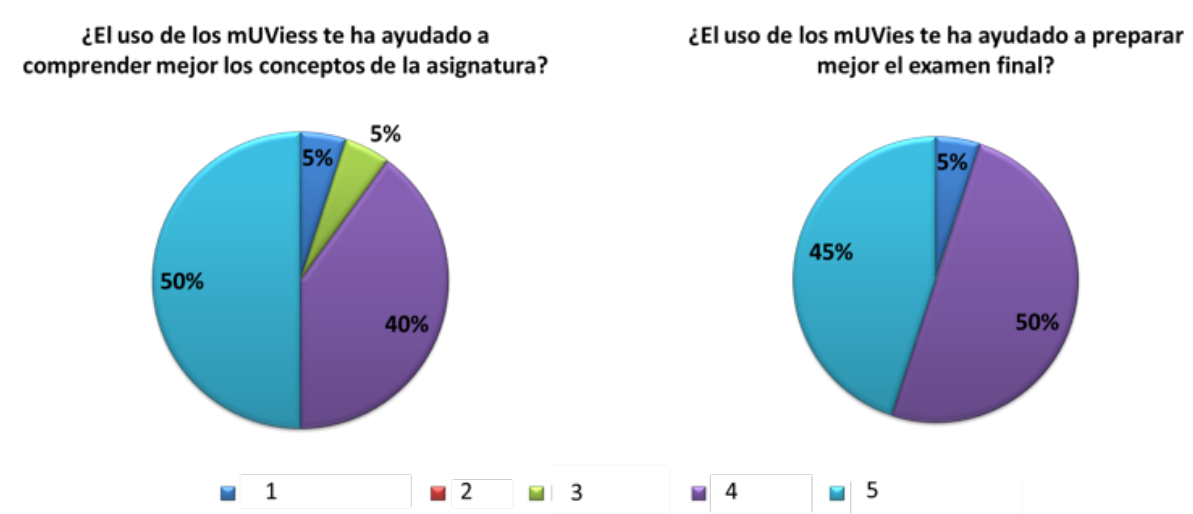

Figura 1. Valoración del alumnado sobre los mUVies
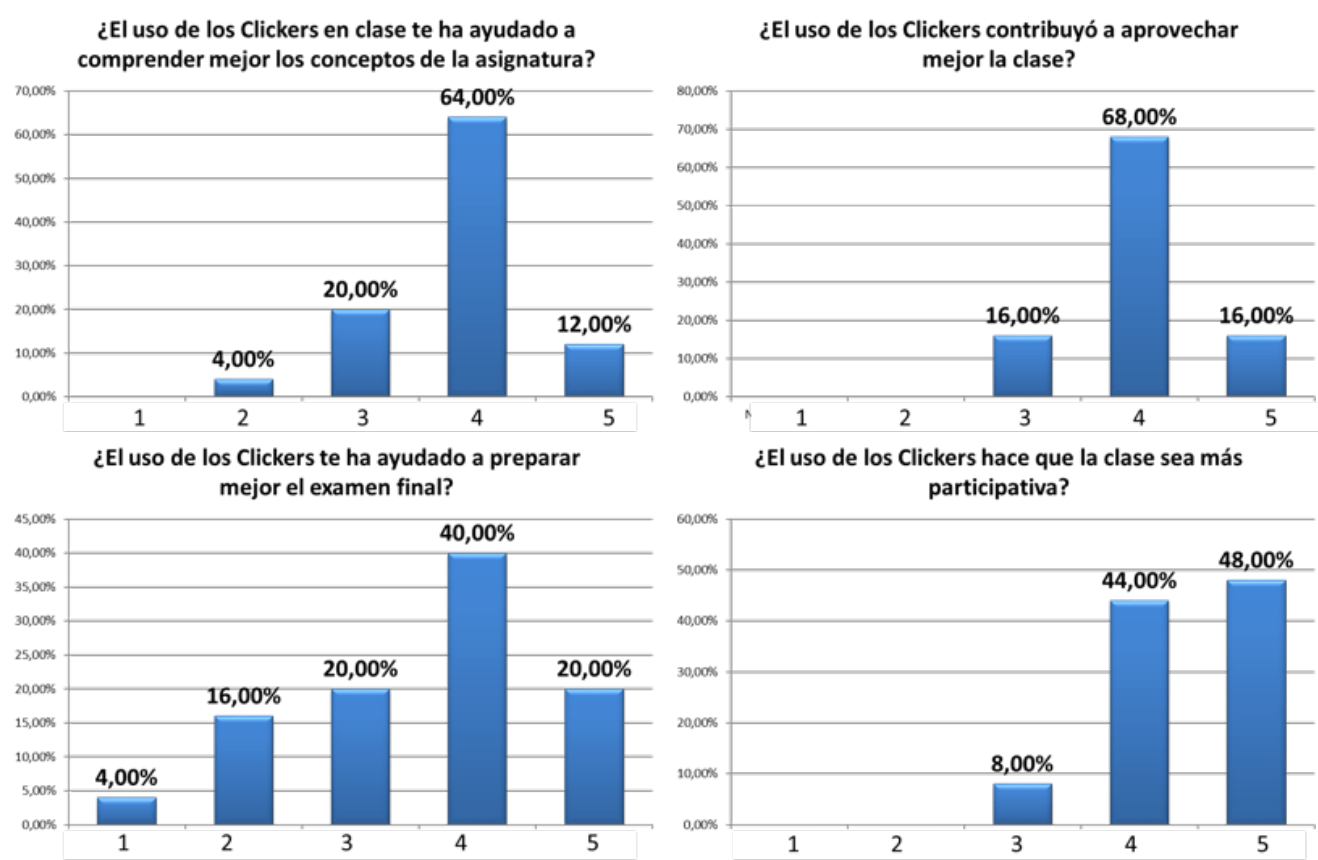

Figura 2. Valoracion del alumnado sobre el uso de Clickers

Los datos recogidos en los mismos informan de que la acogida por parte de los estudiantes del uso de los Clickers fue muy positiva, pues los niveles más desfavorables (1 y 2) fueron seleccionados en las cuatro cuestiones en un porcentaje nunca superior al $20 \%$. Por contra, el nivel 4 contó con un porcentaje nunca inferior al $40 \%$ y si, además, se agregan los porcentajes de este nivel y el siguiente (5) se obtiene que más del 70\% del alumnado percibió que el uso de Clickers les ayudó a comprender mejor la materia y a aprovechar la clase, mientras que para un $60 \%$ ha ayudado a preparar mejor el examen. Asimismo, más del $90 \%$ consideraron que estos elementos hacían la clase más participativa.

Por otra parte, los datos respecto al uso de los mUVies son todavía más favorables. En el caso de esta herramienta, el porcentaje de estudiantes que la considera positiva 
-en cuanto a la repercusión que la misma tuvo en la mejora de comprensión de la materia, así como en la preparación del examen final- es muy superior a la opinión sobre los mismos ítems con respecto a los Clickers. Concretamente, el porcentaje de estudiantes que seleccionaron el nivel 4 o 5 para los mUVies fue del $90 \%$ para la primera y del $95 \%$ para la segunda pregunta, frente al $76 \%$ y el $92 \%$, respectivamente, para las mismas cuestiones en el caso de la herramienta Clickers.

En cuanto a la valoración que los estudiantes dieron al uso de ambas herramientas (escala de 0 a 10), la Tabla 1 recoge las distribuciones de frecuencias correspondientes:

Tabla 1. Valoración del alumnado sobre el uso de Clickers y mUVies

\begin{tabular}{lcclcc}
\hline $\begin{array}{l}\text { Valoración } \\
\text { uso Clickers }\end{array}$ & $\mathrm{fi}$ & $\mathrm{Fi}$ & $\begin{array}{l}\text { Valoración } \\
\text { uso mUVies }\end{array}$ & $\mathrm{fi}$ & $\mathrm{Fi}$ \\
\hline 0 & $0 \%$ & $0 \%$ & 0 & $0 \%$ & $0 \%$ \\
1 & $0 \%$ & $0 \%$ & 1 & $0 \%$ & $0 \%$ \\
2 & $0 \%$ & $0 \%$ & 2 & $0 \%$ & $0 \%$ \\
3 & $0 \%$ & $0 \%$ & 3 & $0 \%$ & $0 \%$ \\
4 & $0 \%$ & $0 \%$ & 4 & $0 \%$ & $0 \%$ \\
5 & $4.17 \%$ & $4.17 \%$ & 5 & $5.26 \%$ & $5.26 \%$ \\
6 & $4.17 \%$ & $8.33 \%$ & 6 & $0 \%$ & $5.26 \%$ \\
7 & $25 \%$ & $33.33 \%$ & 7 & $5.26 \%$ & $10.53 \%$ \\
8 & $20 \%$ & $54.17 \%$ & 8 & $15.79 \%$ & $26.32 \%$ \\
9 & $37.50 \%$ & $91.67 \%$ & 9 & $21.05 \%$ & $47.37 \%$ \\
10 & $8.33 \%$ & $100 \%$ & 10 & $52.63 \%$ & $100 \%$ \\
\hline
\end{tabular}

En ningún caso se ha dado una puntuación inferior a 5 en el uso de ambas tecnologías, y la proporción de calificaciones superiores al 7 (notable) es superior al $90 \%$ en ambos casos. Si se consideran las valoraciones dadas a los Clickers debemos decir que:

- Un $45.83 \%$ del alumnado da una valoración igual o superior a 9

- En el $66.67 \%$ de los casos la puntuación es igual o superior a 8

- El $91.67 \%$ de los estudiantes les asigna una valoración igual o superior a 7

Y en el caso de los mUVies dichos porcentajes son:

- Un 73.68\% de los estudiantes otorga una puntuación igual o superior al 9

- El $89.47 \%$ lo valoran con nota igual o superior a 8

- Un $94.74 \%$ asignan una puntuación igual o superior de 7

Además, puede observarse como también en la cuestión referente a la valoración, los mUVies obtienen mejores resultados que los Clickers, no sólo en términos porcentuales tal y como acaba de exponerse, sino que además el valor medio es mayor. A partir de los datos contenidos en la Tabla 1 se obtiene que los Clickers recibieron una valoración media de 8.08 puntos, mientras que la de los mUVies fue de 9.05, es decir que hay casi un punto de diferencia entre ambos. La puntuación máxima (un 10) se da en un porcentaje elevado de casos para mUVies mientras que para los Clickers la valoración modal (la más repetida) es un 9.

\subsection{Análisis de las variables de carácter objetivo}

Tal y como se indicó, se han considerado como variables de tipo objetivo las relacionadas con el rendimiento académico del estudiante, esto es, su nota de evaluación 
continua y la del examen final de la asignatura. El análisis de las mismas, así como su posible relación con la valoración que el estudiantado hizo sobre el uso de las TIC consideradas, se aborda inicialmente, mediante el uso del diagrama Box-Whisker, que permite comprobar la existencia de dos valores extremos o alejados de la media para las variables "Nota de Evaluación continua" y "Nota de examen final", datos que se eliminan del estudio.

Tras su exclusión, puede comprobarse que en ningún caso las variables consideradas han tomado un valor inferior a cinco, con mejores resultados en el examen final que en la evaluación continua.

Concretamente, los valores medios para dichas características, así como algunas medidas de posición relevantes, se recogen en la Tabla 2.

Tabla 2. Medidas de posición de las valoraciones según herramienta y nota

\begin{tabular}{lcccc}
\hline & $\begin{array}{c}\text { Valoración uso } \\
\text { Clickers }\end{array}$ & $\begin{array}{c}\text { Valoración uso } \\
\text { mUVies }\end{array}$ & $\begin{array}{c}\text { Nota evaluación } \\
\text { continua }\end{array}$ & Nota examen \\
\hline Valoración media & 8.14 & 8.94 & 8.10 & 8.54 \\
Desviación típica & 1.28 & 1.39 & 1.26 & 1.12 \\
Coef. var. de Pearson & 0.16 & 0.16 & 0.16 & 0.13 \\
Mínimo & 5 & 5 & 5.64 & 5.57 \\
Máximo & 10 & 10 & 10 & 9.75 \\
Sesgo Estandarizado & -1.38 & -2.72 & -1.21 & -2 \\
Curtosis Estandarizada & 0.13 & 2.41 & -0.34 & 0.47 \\
\hline
\end{tabular}

Se constata aquí que las valoraciones y calificaciones medias son muy altas (superiores al 8 en todos los casos), con una representatividad más que aceptable dado el valor del coeficiente de variación de Pearson (inferior al 17\% para las cuatro características consideradas).

En cuanto a las variables relacionadas con el rendimiento académico del alumnado, cabe indicar que se han obtenido, por regla general, mejores resultados en el examen final que en la evaluación continua. Este hecho podría explicar que estando muy bien valoradas por el estudiantado las dos herramientas tecnológicas se haya otorgado mayor puntuación al uso de los mUVies. Debe tenerse en cuenta que, a diferencia de los Clickers, los mUVies han estado a disposición del alumnado fuera del aula y han sido visitados, por término medio en más de 450 ocasiones, tal y como registra el contador de visitas de la plataforma Mmedia (https://mmedia.uv.es).

Esta situación evidencia que los mUVies han sido un material de apoyo importante para el estudiante de cara a la preparación del examen final, mientras que los Clickers han servido, fundamentalmente, para realizar un seguimiento del curso, detectando puntos fuertes y débiles que han sido potenciados y corregidos tanto por el profesorado como por el alumnado de cara al examen final, pero no han supuesto un material de apoyo tan potente para el proceso de aprendizaje como los vídeos docentes universitarios. En este sentido cabe preguntarse si hay relación entre las cuatro características consideradas, pretendiendo con ello dar respuesta a cuestiones como: ¿existe relación entre la valoración que el estudiante da a los Clickers y a los mUVies?; ¿están relacionadas dichas valoraciones con el rendimiento académico del alumnado?; esto es, por regla general, si un estudiante valora bien/mal el uso de los Clickers, ¿valora en el mismo sentido el uso de los mUVies?; ¿el alumnado con una buena percepción del uso de las tecnologías (Clickers/mUVies) obtuvieron un buen rendimiento académico? Para dicho estudio utilizamos la matriz de correlación presentada en la Tabla 3.

Podemos concluir que, aunque la relación es positiva entre cada par de variables consideradas, a diferencia de lo que cabía esperar, el coeficiente de correlación en todos los casos excepto entre la nota de evaluación continua y la nota del examen está 
Tabla 3. Matriz de correlación entre valoraciones según herramienta y nota

\begin{tabular}{lcccc}
\hline & $\begin{array}{c}\text { Valoración uso } \\
\text { Clickers }\end{array}$ & $\begin{array}{c}\text { Valoración uso } \\
\text { mUVies }\end{array}$ & $\begin{array}{c}\text { Nota evaluación } \\
\text { continua }\end{array}$ & Nota examen \\
\hline Valoración uso Clickers & 1 & 0.099 & 0.092 & 0.149 \\
$\begin{array}{l}\text { Valoración uso mUVies } \\
\begin{array}{l}\text { Nota evaluación } \\
\text { continua }\end{array}\end{array}$ & 0.099 & 1 & 0.021 & 0.093 \\
Nota examen & 0.092 & 0.021 & 1 & 0.643 \\
\hline
\end{tabular}

próximo a cero. Esto indica que no hay relación entre la percepción que el alumnado tuvo del uso de Clickers y los vídeos docentes y su rendimiento académico.

Por otra parte, los valores correspondientes al "sesgo estandarizado" y la "curtosis estandarizada" contenidos en la Tabla 2 permiten intuir el cumplimiento de la hipótesis de Normalidad para las variables consideradas, excepto para la valoración que recibieron los mUVies. Adicionalmente, los gráficos de probabilidad Normal (Figura 3 y Figura 4) refuerzan esa intuición, ya que los datos no se encuentran "muy alejados" de la recta.

\section{Clickers}

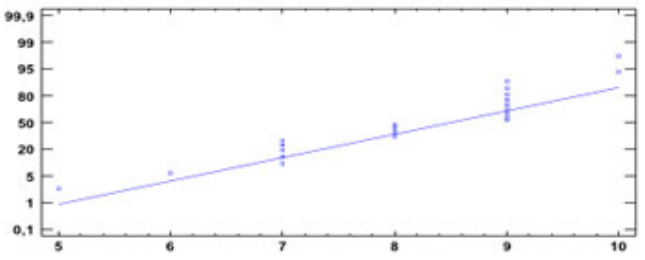

mUVies

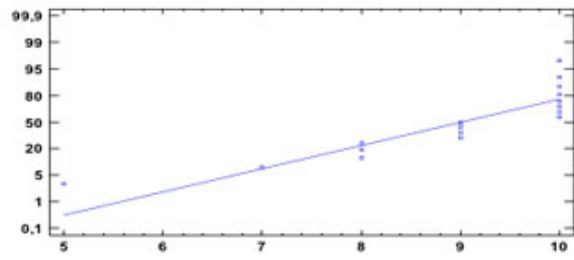

Figura 3. Gráfico de probabilidad Normal para variables subjetivas

Nota evaluación continua

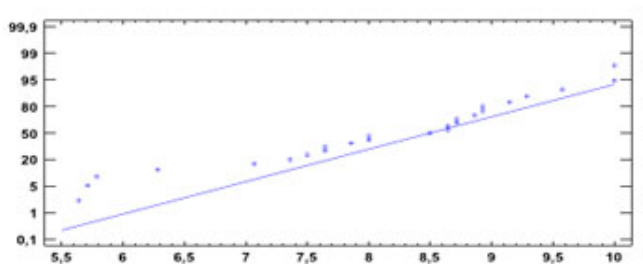

Nota examen

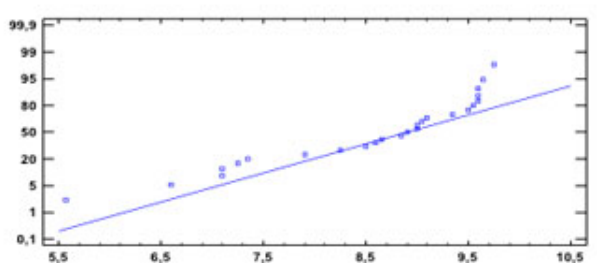

Figura 4. Gráfico de probabilidad Normal para variables objetivas

Con la finalidad de completar el estudio inferencial, se hace uso de test de Kolmogorov-Smirnov (véase Tabla 4).

Así, al poder asumirse la Normalidad para las variables "valoración uso de Clickers", y las notas de evaluación continua y examen final, son de aplicación las propiedades del modelo Normal. Tomando los mismos parámetros muestrales como estimadores de los parámetros media y varianza poblacionales y según los valores contenidos en la Tabla 4 se obtiene que, a nivel de toda la población de estudiantes, el $99.73 \%$ de ellos:

- Valorarían el uso de los Clickers entre un 4.3 y un 10

- Obtendrían una nota de evaluación continua entre un 4.32 y un 10

- Obtendrían entre un 5.18 y un 10 en el examen final 
Tabla 4. Test Kolmogorov-Smirnov. Valoraciones según herramienta y notas

\begin{tabular}{|c|c|c|c|c|c|c|c|}
\hline & \multicolumn{2}{|c|}{ Parámetros normales ${ }^{a, b}$} & \multicolumn{3}{|c|}{ Máximas diferencias extremas } & \multirow[b]{2}{*}{$\begin{array}{c}\text { Estadístico } \\
\text { de prueba }\end{array}$} & \multirow[b]{2}{*}{$\begin{array}{l}\text { Sig. Asintótica } \\
\text { (bilateral) }\end{array}$} \\
\hline & Media & $\begin{array}{l}\text { Desviación } \\
\text { típica }\end{array}$ & Absoluto & Positivo & Negativo & & \\
\hline Clickers & 8.14 & 1.28 & 0.249 & 0.16 & -0.249 & 0.249 & $0.01^{c}$ \\
\hline mUVies & 8.94 & 1.39 & 0.247 & 0.223 & -0.247 & 0.247 & $0.007^{c}$ \\
\hline $\begin{array}{l}\text { Nota } \\
\text { evaluación } \\
\text { continua }\end{array}$ & 8.1 & 1.26 & 0.147 & 0.087 & -0.147 & 0.147 & $0.169^{c}$ \\
\hline Nota examen & 8.54 & 1.12 & 0.171 & 0.139 & -0.171 & 0.171 & $0.058^{c}$ \\
\hline
\end{tabular}

${ }^{a}$ La distribución de prueba es Normal; ${ }^{b}$ Se calcula a partir de los datos; ${ }^{c}$ Corrección de significación de Lilliefors

Estos pueden considerarse unos resultados muy buenos, pues en casi el $100 \%$ de los casos la valoración de las herramientas/puntuación de las notas sería superior a 4 (a 5 en caso de la calificación en el examen final) y la máxima valoración/puntuación a alcanzar sería un 10.

\subsection{Volumen de trabajo del profesorado y el estudiantado}

La incorporación de cualquier tecnología en el proceso de enseñanza-aprendizaje suele repercutir en un esfuerzo adicional tanto por parte del docente, que ve como se incrementa su volumen de trabajo habitual, como del estudiantado que debe familiarizarse con las herramientas desde un punto de vista académico y, además, ve aumentado su trabajo, especialmente fuera del aula. Así, en el presente epígrafe se pretende estimar el tiempo que tanto el equipo docente de la asignatura como el estudiantado tuvo que dedicar a la implantación y al uso tanto de Clickers como de mUVies. En el caso del volumen de trabajo del profesorado cabe realizar un inciso: la estimación que se realiza es válida para el primer curso de la implantación de las herramientas, esto es, partiendo de "cero", y comprende el estudio de manuales, elaboración de diapositivas, tiempo de grabación, etc. Este esfuerzo inicial, en principio, se rentabilizará con el tiempo, pues en cursos sucesivos deberían ser necesarias sólo modificaciones que permitieran mejorar los materiales, no suponiendo, por tanto, un volumen de trabajo tan elevado como el derivado de su puesta en marcha el primer curso. Dichas estimaciones se recogen en la Tabla 5 atendiendo a la tecnología educativa (Clickers o mUVies) utilizada en cada caso.

Tabla 5. Cómputo de volumen de trabajo del profesorado

\begin{tabular}{|c|c|c|c|c|c|}
\hline & \multicolumn{2}{|c|}{ Clickers } & \multicolumn{3}{|c|}{ mUVies } \\
\hline & $\begin{array}{l}\text { Conocimiento } \\
\text { adecuado } \\
\text { herramienta }\end{array}$ & $\begin{array}{l}\text { Elaboración } \\
\text { sondeo }\end{array}$ & Elaboración material/ vídeo & Grabación vídeo & Revisión, metadatos \\
\hline Dedicación & $50 \mathrm{~h}$ & $5 \mathrm{~h}$ & $2 \mathrm{~h}$ & $2 \mathrm{~h}$ & $0.5 \mathrm{~h}$ \\
\hline Número & & $3 h$ & $15 \mathrm{~h}$ & $15 \mathrm{~h}$ & $15 \mathrm{~h}$ \\
\hline Subtotal & $50 \mathrm{~h}$ & $15 \mathrm{~h}$ & $30 \mathrm{~h}$ & $30 h$ & $7.5 \mathrm{~h}$ \\
\hline TOTAL & \multicolumn{2}{|c|}{$65 \mathrm{~h}$} & \multicolumn{3}{|c|}{$67.5 \mathrm{~h}$} \\
\hline
\end{tabular}

Para la elaboración de la Tabla 5 se ha tenido en cuenta que, en el caso de la puesta en marcha de los Clickers, fue necesario:

- El estudio de un manual del software TurningPoint, necesario para la realización de los diferentes sondeos, a lo que se dedicó no menos de 50 horas de trabajo. 
- La realización de tres sondeos de Clickers en clase. Para la confección de las preguntas de cada sondeo y elaboración del material correspondiente se hizo uso de unas cinco horas, aproximadamente, por sondeo.

Así, el cómputo del volumen de trabajo para el profesorado, derivado del uso de los Clickers, fue de 65 horas.

En cuanto a la elaboración de los mUVies, debe indicarse que la ayuda del soporte técnico del SFPIE de la Universitat de València, disminuyó considerablemente el volumen de trabajo asociado a cada vídeo educativo. En cualquier caso, la tarea asociada a su realización por parte del equipo docente, constaba de tres fases claramente diferenciadas:

- Elaboración del material necesario para la grabación.

- La grabación de los video tutoriales en sí.

- Revisión de los vídeos, una vez que los técnicos habían procedido al montaje del mismo, e introducción de metadatos.

La dedicación estimada para las dos primeras tareas fue de unas dos horas por fase para cada vídeo docente, mientras que para la tercera fue de una media hora. Esto hace que a cada uno de ellos se dedicaran cuatro horas y media. Si se tiene en cuenta que se elaboraron 15 vídeos, el cómputo del volumen del profesorado asociado a esta herramienta fue de 67 horas y media. Por lo tanto, si se tiene en cuenta la estimación de dedicación global a ambas tecnologías fueron necesarias 132.5 horas, es decir, más de dieciséis jornadas laborales (de 8 horas) completas, o equivalentemente, más de tres semanas laborales a tiempo completo.

Por otra parte, debe tenerse en cuenta que son dos, profesorado y estudiantado, los agentes involucrados en el proceso enseñanza-aprendizaje y que, como en cualquier proceso, para optimizar los resultados es necesario que la dedicación del alumnado sea acorde a la del profesorado. Por ello, se procede a estimar, también, el volumen de trabajo que supuso para el alumnado el uso de las dos TIC. Para llevar a cabo dicha estimación se hará uso de las respuestas a la cuestión planteada en el cuestionario sobre el número de veces que había necesitado el estudiante visionar el video hasta su comprensión. Así se obtendrá, no sólo la estimación buscada, sino que se podrá analizar la adecuación de los vídeos educativos. En efecto parece que, si el estudiante necesitó una media de visionados cercana a la unidad, esto es, si por término medio comprendió el contenido del vídeotutorial "a la primera", se estaría en el camino correcto. Un análisis descriptivo de los datos recogidos a través del cuestionario evidencia que en un $84.21 \%$ de los casos ha sido necesaria la visualización del mUVie un máximo de 2 veces para su comprensión.

La obtención, por otra parte, de algunas medidas de posición relevantes, como la media (=1.58), mediana (=1) y moda (=1) de la distribución de frecuencias de dichos datos, permite concluir que por término medio ha hecho falta visualizar cada vídeo menos de dos veces, que en el $50 \%$ de las ocasiones ha sido necesaria tan solo una visualización y que la respuesta más repetida a la pregunta planteada ha sido: "he necesitado visualizar el mUVie una vez", esto es, que se ha entendido a la primera.

A partir de lo indicado, es posible estimar la dedicación del alumnado, esto es, el tiempo que destinó al uso de las herramientas consideradas. Así, en la Tabla 6 se recoge la estimación atendiendo a la tecnología utilizada (Clickers o mUVie).

Para su elaboración, se ha tenido en cuenta que:

- El tiempo dedicado a los sondeos se ha ceñido únicamente a la duración del mismo, esto es, 0.5 horas en cada sondeo. Este supuesto se sustenta en que se está 
Tabla 6. Cómputo de la dedicación del alumnado

\begin{tabular}{lccc}
\hline & \multicolumn{2}{c}{ Clickers } & \multicolumn{2}{c}{ mUVies } \\
\cline { 2 - 3 } & Realización sondeo & $N^{\circ}$ visualizaciones / vídeo & Tiempo/ vídeo \\
\hline Dedicación & $0.5 \mathrm{~h}$ & 1.58 & $15 \mathrm{~min}$ \\
Número & 3 & 15 & \\
Subtotal & $1.5 \mathrm{~h}$ & 23.7 visualizaciones & $355.5 \mathrm{~min} \approx 6 \mathrm{~h}$ \\
TOTAL & $1.5 \mathrm{~h}$ & & $6 \mathrm{~h}$ \\
\hline
\end{tabular}

analizando el volumen de trabajo añadido que ha significado para el alumnado la incorporación de los Clickers, por lo que, al no existir prerrequisito para contestar al sondeo, no se tiene en cuenta el tiempo de estudio fuera del aula que el estudiante haya utilizado para contestar a las preguntas, al ser supuestamente el mismo que en principio debería haber dedicado para "llevar la materia al día".

Así, el cómputo del volumen de trabajo para el alumnado, derivado del uso de los Clickers en los tres sondeos realizados, fue de una hora y media.

- En cuanto al uso de los mUVies, atendiendo a que por término medio fueron necesarias 1.58 visualizaciones por vídeo, que la duración media de los mismos es de 15 minutos y que debían visualizar 15, el cómputo del volumen asociado a esta herramienta fue de 355.5 minutos, esto es, aproximadamente 6 horas.

Por lo tanto, si se tiene en cuenta la estimación de la dedicación global de ambas tecnologías, fueron necesarias 7 horas y media, es decir, aproximadamente un día laboral (de 8 horas).

\section{CONCLUSIONES Y DISCUSIÓN}

El plan de estudios del grado GIB de la Universitat de València ha presentado desde su implantación, un problema derivado del escaso número de horas docentes dedicadas a la asignatura de Estadística. En efecto, el contenido de la asignatura (descriptiva, probabilidad e inferencia) necesitaría para su correcta impartición/asimilación un volumen mayor de horas o créditos. Para intentar dar solución al problema planteado, a lo largo del curso 2017-2018, el equipo docente de la misma decidió incorporar en la metodología dos herramientas propias del Flipped Classroom: una herramienta de respuesta de audiencia (los Clickers) y vídeos elaborados con el soporte técnico del SFPIE (denominados mUVie).

Con la finalidad de analizar la percepción del alumnado, así como el volumen de trabajo generado por la incorporación de las TIC citadas (tanto para el profesorado como para el alumnado) se procedió a recabar información a través de un cuestionario $a d$-hoc con diferentes tipos de preguntas (de escala Likert a 5 niveles, de valoración -escala 0 a 10- acerca del uso de ambas herramientas, etc.).

El análisis de los datos obtenidos a partir del mismo ha permitido obtener las siguientes conclusiones.

Primero, la acogida por parte del alumnado ha sido más que satisfactoria. En efecto, el porcentaje de estudiantes que indicaban estar "bastante" o "muy" satisfechos con el uso de ambas herramientas fue superior al 70\% y alcanzó niveles superiores al 90\% en lo relativo al incremento en la participación del alumnado en el aula. La calificación (entre 0 y 10) otorgada por los estudiantes superó, por término medio, el notable alto, llegando a ser de sobresaliente para los vídeos educativos. En la valoración subjetiva, los video tutoriales recibieron una mejor acogida que los Clickers. A modo de 
ejemplo cabe citar que mientras que el $45.83 \%$ de los encuestados otorgó una valoración superior o igual a 9 a los Clickers, el porcentaje para esa calificación ascendió al 73.68\% en el caso de los vídeos.

Esta mejor aceptación de los vídeos puede estar relacionada con que la forma de evaluación de la asignatura en el examen final no se lleva a cabo mediante preguntas de tipo test, sino con la resolución de ejercicios prácticos, de modo que los videos suelen ajustarse mejor a la forma de evaluar que el uso de la HRA. En Barac y PardoGarcía (2015) analizan las diferencias de aceptación de los Clickers en una asignatura cuantitativa respecto a otra de carácter más teórico obteniendo mejores resultados en la segunda. Concretamente en la asignatura más teórica la valoración de "aclarar conceptos previamente explicados" dominaba frente a "hacer autoevaluación", al contrario que en la asignatura de carácter cuantitativo. Adicionalmente, siguiendo la literatura analizada, León, Peñalba y Escudero señalan que:

Estas observaciones tienen en su base el apoyo que diferentes trabajos han ofrecido la idea de que determinados análisis de las preguntas y respuestas que se dan el aula permiten conocer el grado en que los sujetos han realizado inferencias de algún tipo y la estructura de conocimiento que, por tanto, poseen. También consideran los efectos que diferentes rasgos de esta interacción pueden tener sobre el propio aprendizaje. (2002, p. 11)

De modo que consideramos que el uso conjunto de ambas herramientas tecnológicas refuerza su utilidad para el aprendizaje, ya que el uso de los Clickers puede mostrar mejor a los estudiantes sus puntos débiles y los conceptos que peor han asimilado o comprendido, y son los vídeos los que les permiten volver a visualizar los contenidos para preparar mejor el examen, tal y como ellos mismos manifiestan en sus respuestas al cuestionario.

Segundo, el cómputo total del volumen de trabajo asociado al uso de las tecnologías consideradas asciende a 7 horas y media en el caso del alumnado, siendo mayor en el caso de los vídeos (6 horas) que en el de los Clickers (1.5 horas). Sin embargo, en lo que respecta al profesorado este estudio arroja un balance mucho más elevado, pues se estima una dedicación de unas 132.5 horas (más de tres semanas laborales a tiempo completo), repartida de una manera casi equitativa entre Clickers (65 horas) y vídeos docentes (67.5 horas). Si bien es cierto que son cifras aproximadas que se espera sean aplicables únicamente al primer curso de implantación de estas tecnologías, ya que en cursos posteriores el volumen de trabajo debería disminuir considerablemente y estar asociado, básicamente, a los reajustes que se deriven de la mejora y optimización de los materiales elaborados.

Tercero, en cuanto a la implicación que esto haya podido tener en el rendimiento académico del alumnado cabe indicar que, aunque este fue muy positivo (la tasa de presentados fue del $94.4 \%$ y la de aprobados sobre presentados del 89.6\%), la matriz de correlación obtenida entre las variables cuantitativas relacionadas con la valoración que el alumnado dio al uso de las TIC y su rendimiento (medido a través de su nota de evaluación continua y la del examen final) no permite confirmar la existencia de dicha relación. La propia matriz de correlación de la Tabla 3 parece indicar que el trabajo constante o la ausencia del mismo, reflejados a través de la nota de evaluación continua parece ser el factor más determinante en los resultados del examen final (Dixon y Rawlings, 1987), de modo que el uso de las herramientas TIC sólo reforzaría el aprendizaje en la medida en que los alumnos estén dispuestos a trabajar.

Hay que resaltar que en cualquier caso, el alumnado otorgó una calificación más que positiva (4.79 sobre 5) a la metodología utilizada en la asignatura, en las encuestas realizadas por la Unitat de Qualitat (servicio universitario encargado de esta medición). 
A modo de conclusión general podemos considerar que, aunque el uso de estas tecnologías ha supuesto un incremento en el volumen de trabajo de los dos agentes implicados en el proceso enseñanza-aprendizaje (17 veces mayor para el profesorado), tanto la percepción del alumnado como los resultados objetivos invitan a recomendar la incorporación de las metodologías docentes de recursos audiovisuales y de herramientas de respuesta de audiencia para la mejora de los resultados y el aprendizaje. Esta recomendación está especialmente indicada para las asignaturas que tienen un carácter cuantitativo. Por otro lado, y aunque recomendamos el uso combinado y complementario de ambas TIC, si el profesor debe elegir la implantación de sólo una de las herramientas por un problema de disponibilidad de tiempo para la preparación de materiales, teniendo en cuenta la acogida y resultados presentados recomendaríamos más los vídeos educativos que los Clickers.

\section{REFERENCIAS}

Badia-Valiente, J., Olmo-Cazevieille, F., y Navarro-Jover, J. (2016). On-line quizzes to evaluate comprehension and integration skills. Journal of Technology and Science Education, 6(2), 75-90. Descargado de http://dx.doi.org/10.3926/jotse.189

Baker, A. (2016). Active Learning with interactive videos: Creating student-guided learning materials. Fournal of Library \& Information Services in Distance Learning, 10(3-4), 79-87. https://doi.org/10.1080/1533290X.2016.1206776

Baker, J. (2000). The "Classroom Flip": Using web course management tools become the guide by the side. Communication Faculty Publications, 15, 9-17. Descargado de http:// digitalcommons.cedarville.edu/media_and_applied_communications_publications/15

Barac, M., y López-Rodríguez, M. (2017). Cuantificando el impacto de los clickers en la docencia universitaria. En ESIC (Ed.), IV Simposio Internacional Innovación Aplicada (ESIC-IMAT) (pp. 59-60). Valencia: ESIC-IMAT.

Barac, M., y Pardo-García, C. (2015). Uso de los clickers en asignaturas con características docentes diferentes: motivación y evaluación del alumnado. IV Jornades IDES (pp. 339-354). Valencia. Descargado de http://www.uv.es/econdocs/ides2015/Llibre_actes_IV_jornades_IDES_2015

Bruff, D. (2009). Teaching with classroom response systems: creating active learning environments. Hoboken: Wiley \& Sons.

Caballer-Tarazona, M., y Pardo-García, C. (2014). Statistics continuous assessment through an activity using an interactive voting system. 6th International Conference on Education and New Learning Technologies (pp. 2939-2944). Barcelona: IATED Academy. Descargado de https://pages.uv.es/piclickers/eng/docs/pedulearn14.pdf

Calvo-Roselló, V., López-Rodríguez, M. I., y Ruiz-Ponce, F. (2017). Uso de un Electronic Voting System: una radiografía del aula universitaria en tiempo real. INNODOCT/17. International Conference on Innovation, Documentation and Education (pp. 715-726). Valencia: Editorial Universitat Politècnica de València.

Casasús, T., Ivars, A., y López-Rodríguez, M. (2018). Present and future of the e-learning in economics schools and faculties. Multidisciplinary fournal for Education, Social and Technological Sciences, 5(1), 44-64. https://doi.org/10.4995/muse.2018.9777

Dixon, R., y Rawlings, G. (1987). Experiences with continuous assessment. Assessment and Evaluation in Higher Education, 12(1), 24-36. https://doi.org/10.1080/0260293870120103

Fuertes, A., García, M., Castaño, M., López, E., Zacares, M., Cobos, M., ... Grimaldo, F. (2016). Uso de herramientas de respuesta de audiencia en la docencia presencial universitaria. Un primer contacto. XXII fornadas sobre la Enseñanza Universitaria de la Informática. JENUI 2016 (pp. 261-268). Descargado de http://hdl.handle.net/2117/90357

Gallardo-Echenique, E. (2012). Hablemos de estudiantes digitales y no de nativos digitales. Universitas Tarraconensis. Revista de Ciències de l'Educació, 1(1), 7-21. Descargado de https://revistes.urv.cat/index.php/ute/article/viewFile/595/574 
García-Aretio, L. (2017). Los MOOC están muy vivos. Respuestas a algunas preguntas. Revista Iberoamericana de Educación a a Distancia, 20(1), 9-27. https://doi.org/10.5944/ried.20.1.17488

Gross, D., Pietri, E., Anderson, G., Moyano-Camihort, K., y Graham, M. (2015). Increased preclass preparation underlies student outcome improvement in the flipped classroom. CBE Life Sciences Education, 14(4), 1-8.

Lage, M., Platt, G., y Treglia, M. (2000). Inverting the Classroom: A Gateway to Creating an Inclusive Learning Environment. The fournal of Economic Education, 31(1), 30-43. Descargado de https://www.jstor.org/stable/1183338

León, J., Peñalba, G., y Escudero, I. (2002). Profe, ¿puedo preguntar? Una breve introducción a la interacción de preguntas y respuestas entre profesor y alumno. Psicología Educativa, 8(2), 107-126. Descargado de https://journals.copmadrid.org/psed/art/83e8ef518174e1eb6be4a0778d050c9d

López-Rodríguez, M., y Barac, M. (2016). Uso de los clickers en una asignatura de diseño de experimentos. V fornades IDES. Valencia: España.

López-Rodríguez, M., Palací-López, J., y Palací-López, D. (2015). Use of ICTs in degree studies: a descriptive analysis. 8th International Conference of Education (ICERI), Research and Innovation (pp. 2286-2290). Descargado de https://library.iated.org/publications/ICERI2015

Navarro-Jover, J., y Olmo-Cazevieille, F. (2014). Socrative, una aplicación web 2.0 para evaluar la comprensión de los estudiantes. Fornadas de Innovación Educativa y Docencia en Red de la Universidad Politécnica de Valencia (pp. 69-79). Valencia: Editorial Universitat Politécnica de València.

Perdomo, W. (2016). Estudio de evidencias de aprendizaje significativo en un aula bajo el modelo Flipped Classroom. Edutec. Revista Electrónica de Tecnología Educativa, 55, 1-17.

Pintor-Holguín, E., Gargantilla-Madera, P., Herreros Ruiz-Valdepeñas, B., y López del Hierro, M. (2014). Kahoot en docencia: una alternativa practica a los clickers. XI fornadas Internacionales de Innovación Universitaria. Educar para transformar (pp. 322-329). Descargado de http://hdl.handle.net/11268/3603

Pueo, B., Jimenez-Olmedo, J., Penichet-Tomas, A., y Carbonell-Martinez, J. (2017). Aplicación de la herramienta EDpuzzle en entornos de aprendizaje individuales dentro del aula. En R. Roig-Vila (Ed.), Investigación en docencia universitaria. Diseñando el futuro a partir de la innovación educativa. Barcelona: Octaedro.

Serrano-Pastor, R., y Casanova-López, O. (2017). Acercar la flipped classroom al aula de música universitaria mediante el uso de aplicaciones para realizar y gestionar vídeos. Percepción y valoración de los estudiantes. European fournal of Scientific Research, 13(1), 89-101. http://dx.doi.org/10.19044/esj.2017.v13n1p89

Singh, V., Abdellahi, S., Lou-Maher, M., y Latulipe, C. (2016). The video collaboratory as a learning environment. SIGCSE '16 Proceedings of the 47th ACM Technical Symposium on Computing Science Education (pp. 352-357). Descargado de http://dx.doi.org/10.1145/2839509.2844588 